\title{
Association between severity of prenatally diagnosed hydronephrosis and receipt of surgical intervention postnatally among patients seen at a fetal-maternal center
}

Zoë G. Baker ${ }^{1 *}(1)$, Arthi Hannallah', Melissa Trabold ${ }^{1}$, Danielle Estell ${ }^{2}$, Cherry Deng ${ }^{3}$, Andy Y. Chang ${ }^{1,2}$, S. Scott Sparks ${ }^{1,2}$, Roger De Filippo ${ }^{1,2}$ and Evalynn Vasquez ${ }^{1,2}$

\begin{abstract}
Background: Hydronephrosis (HN) is the most common abnormality detected on prenatal ultrasound. This study sought to stratify outcomes of patients by severity of prenatal HN with postnatal outcomes.

Methods: This was a retrospective review of patients referred to a tertiary care fetal-maternal clinic with diagnosis of prenatal HN from 2004 to 2019. HN severity was categorized as mild, moderate, or severe. Data were analyzed to determine the association between HN severity and surgical intervention. Decision for surgery was based on factors including history of multiple urinary tract infections, evidence of renal scarring, and/or reduced renal function. Surgery-free survival time was represented by the Kaplan-Meier method, and hazard ratios were calculated using the log-rank test.

Results: 131 kidneys among 101 infants were prenatally diagnosed with hydronephrosis; 35.9\% had mild HN, 29.0\% had moderate HN, and $35.1 \%$ had severe HN. $8.5 \%$ of patients with mild HN, $26.3 \%$ of patients with moderate HN, and $65.2 \%$ of patients with severe HN required surgery. Patients with severe $\mathrm{HN}$ were $12.2(95 \% \mathrm{Cl} 6.1-24.4 ; p<0.001)$ times more likely to undergo surgery for $\mathrm{HN}$ than patients with mild $\mathrm{HN}$ and $2.9(95 \% \mathrm{Cl} 1.5-5.3 ; p=0.003)$ times more likely to undergo surgery than patients with moderate HN. Patients with moderate HN were 4.3 times more likely to require surgery than patients with mild $\mathrm{HN}(95 \% \mathrm{Cl} 1.5-12.9 ; p=0.01)$. Median age at surgery was 11.8 months among patients with mild HN (IQR 11.7-14.1 months), 6.6 months among patients with moderate HN (IQR 4.2-16.4 months), and 5.4 months among patients with severe HN (3.7-12.4 months).

Conclusion: Among this cohort of referrals from a fetal-maternal clinic, severity of HN correlated with increased likelihood of surgical intervention. Continued assessment of patients with prenatal HN should be evaluated to best determine the role of the pediatric urologist in cases of prenatal HN.
\end{abstract}

Keywords: Hydronephrosis, Prenatal, Surgery, Fetal, Diagnosis

*Correspondence: zobaker@chla.usc.edu

${ }^{1}$ Division of Urology, Children's Hospital Los Angeles, Los Angeles, CA, USA

Full list of author information is available at the end of the article

\section{Background}

Hydronephrosis $(\mathrm{HN})$ is a descriptive term for dilation of one or both kidneys. It is the most common prenatal abnormality detected by ultrasound [1] and is diagnosed in one to five percent of pregnancies [2, 3]. Increased prenatal screening has enabled physicians to diagnose 
prenatal HN more readily. Renal ultrasonography (US) and voiding cystourethrogram (VCUG) are often performed postnatally for physicians to assess renal function and consider treatment plans.

The Society for Fetal Urology (SFU) has categorized the severity of HN into grades 1-4. This system takes into consideration the degree of pelvic dilation, presence of calyceal dilation, and thinning of parenchymal tissue in the kidneys. Despite advances in prenatal ultrasound screening for $\mathrm{HN}$, there is still some ambiguity in criteria for diagnosing severity and appropriate management. There is debate about the cutoff values for the SFU grading system, though efforts have been made by various professional groups to consolidate findings into a more comprehensible management guide for practicing urologists [4]. Another highly contested matter is the threshold at which surgical intervention is needed. Gender, age, and the presence of urological comorbidities are factors that must also be considered during the course of treatment.

After $\mathrm{HN}$ is detected on prenatal ultrasound, prenatal counseling with pediatric urologists is encouraged in order to form appropriate management plans. Urologists may recommend newborns with prenatal $\mathrm{HN}$ to be put on antibiotic prophylaxis in order to reduce the risk of urinary tract infections (UTIs) [5]. This is usually followed by monitoring with ultrasounds to assess the progression and potential resolution of HN. While current literature indicates that a large proportion of cases are stabilized or resolved following conservative management $[1,3,6]$, studies have reported differing findings regarding the proportion of patients who go on to require surgery. A recent review found that the rate of complete resolution of prenatally-detected $\mathrm{HN}$ in the absence of surgery may range from 33 to 70\% [7]. Discrepancies in the rates of patients who require surgery may be largely due to differences in $\mathrm{HN}$ severity between populations [8-13]. Variation in the proportion of patients with prenatally diagnosed $\mathrm{HN}$ who require surgery after birth may result in difficulty in counseling pregnant women on their child's expected care trajectory. In some patients with HN, surgery may be indicated to prevent or manage recurrent UTIs, kidney dysfunction, ureteropelvic junction obstruction (UPJO) and/or vesicoureteral reflux (VUR) [1, 6-8, 13]. Common surgical interventions for $\mathrm{HN}$, depending on the etiology of hydronephrosis, include pyeloplasties for UPJO, ureteral reimplantations for VUR, and in some cases, nephrectomies for symptomatic, nonfunctional kidneys [7, 9, 13, 14].

The aim of this study is to assess the number and proportion of patients diagnosed with mild (SFU Grade 1 or 2), moderate (SFU Grade 3), and severe (SFU Grade 4) prenatal $\mathrm{HN}$ at a fetal-maternal center that progress to surgery, and to assess the correlation between severity of prenatal diagnosis and probability of postnatal surgical intervention. Time to surgery by prenatal severity score will also be assessed. The results of this study may assist providers in delivering more accurate prenatal counseling to parents following identification of $\mathrm{HN}$, illustrate the importance of prenatal visits, and may also aid pediatric urologists in taking appropriate precautions while identifying the etiology among pediatric patients with $\mathrm{HN}$.

\section{Methods}

Electronic medical records were reviewed among patients who received prenatal HN diagnoses by neonatologists and referred to pediatric urologists at a fetalmaternal center in a large urban setting between 2004 and 2019. Information on prenatal urologic diagnoses and severity of hydronephrosis was recorded from mothers' pediatric urology notes, radiology reports, and/or radiology images. Patients' demographic, clinical, and surgical information were similarly recorded and linked to their mothers' data.

Patients were included in the study if severity of $\mathrm{HN}$ was recorded at the prenatal visit, they attended at least one urology appointment postnatally, and they were at least 12 months old at the time of data analysis. Patients with prenatal anteroposterior (AP) diameters of less than $10 \mathrm{~mm}$ were excluded, as this was the cut-off for patients to receive pediatric urology referrals. Patients were also excluded if they did not survive to term or were less than 12 months old at the time of data analysis. Patients were followed until the date of their surgical intervention, until the date their $\mathrm{HN}$ resolved, or until the date of their last follow-up with a pediatric urologist, up until age 6 years. Prenatal images were reviewed and hydronephrosis severity was assessed by each patient's pediatric urologist. HN severity was classified as mild (SFU 1-2), moderate (SFU 3), or severe (SFU 4). Classifications were based on the status of the calices, extent of pelvic dilation, and presence of parenchymal atrophy. SFU grades of 1 and 2 were combined as some prenatal notes did not differentiate between the two and instead indicated "SFU grade 1/2." Data on both kidneys were recorded among patients prenatally diagnosed with bilateral $\mathrm{HN}$.

The probability of undergoing surgical intervention for $\mathrm{HN}$ was stratified by prenatally diagnosed HN severity. Patients' diagnoses were classified postnatally. If a specific diagnosis was not given due to the transient nature of the patient's HN, it was classified as transient. Decision for surgical intervention was based on the clinical characteristics of the patient including history of multiple recurrent urinary tract infections, evidence of renal scarring, and/or decreased renal function. Surgical interventions of interest included: pyeloplasty, nephrectomy, 
ureteral reimplantation, ureterostomy, ureterocele incision, and other urologic surgeries related to a diagnosis of $\mathrm{HN}$. Among patients who underwent surgery, age at time of surgery was recorded. Kaplan-Meier curves and estimates with confidence intervals were created to assess the probability of receiving surgery versus proceeding with conservative management over time, stratified by HN severity. Whether patients were discharged from the clinic after resolution of $\mathrm{HN}$ without surgery or were receiving continued follow-up and monitoring for $\mathrm{HN}$ at the time of data analysis was also noted. Patients who did not undergo surgery and who had persistent $\mathrm{HN}$ at the time of data analysis were censored at the time of last clinic follow-up visit. Hazard ratios (HR) were calculated using the log-rank test, to determine differences in probability of surgery between patients with severe versus mild $\mathrm{HN}$, severe versus moderate $\mathrm{HN}$, and moderate versus mild $\mathrm{HN}$. Etiology of $\mathrm{HN}$ by prenatal severity was also reported. Pearson $\mathrm{X}^{2}$ tests were utilized to evaluate whether there were any differences in probability of receiving surgery by patient gender, race/ethnicity, primary language, medical insurance type (public versus private), or vesicoureteral reflux (VUR) presence.

This study was reviewed and approved by the institutional review board at Children's Hospital Los Angeles.

\section{Results}

\section{Overall results}

This study included 101 infants and 131 kidneys prenatally diagnosed with HN (Table 1). Mean gestational age at first detection of hydronephrosis was 23.5 weeks (IQR 20-31), though mothers were not referred to pediatric urology until AP diameter was at least $10 \mathrm{~mm}$. There were no significant differences in gestational age at $\mathrm{HN}$ detection by $\mathrm{HN}$ severity. Postnatally, patients were followed for a median of 1.2 years (IQR $0.8-2.3$ years). The majority of patients were male $(\mathrm{n}=75,74.3 \%)$, and $40 \%$ $(\mathrm{n}=41)$ identified as Hispanic. Approximately $70 \%$ of patients were diagnosed with unilateral $\mathrm{HN}(\mathrm{n}=71)$, and $30 \%$ with bilateral $\mathrm{HN}(\mathrm{n}=30)$. Overall, 33.6\% $(\mathrm{n}=44)$ of hydronephrotic kidneys required surgical intervention, including $8.5 \%$ of kidneys diagnosed with mild $\mathrm{HN}$, $26.3 \%$ of those diagnosed with moderate $\mathrm{HN}$, and $65.2 \%$ of those diagnosed with severe HN (Table 2). Of the remaining kidneys, 30 (22.9\%) spontaneously resolved, and $57(43.5 \%)$ did not yet require surgical intervention and were being conservatively managed with serial ultrasound.

The likelihood of requiring surgery varied significantly by prenatal $\mathrm{HN}$ severity. Additionally, among those patients who required surgery, age at surgery varied by $\mathrm{HN}$ severity; patients with moderate and severe $\mathrm{HN}$ generally underwent surgery at younger ages than patients
Table 1 Demographic and prenatal factors among patients prenatally diagnosed with hydronephrosis at fetal-maternal center and followed post-birth from 2004-2019 ( $n=101)$

\begin{tabular}{lcc}
\hline Demographic/prenatal factor & $\mathrm{n}$ & $\%$ \\
\hline Gender & 75 & 74.3 \\
Male & 26 & 25.7 \\
Female & & \\
Race/ethnicity & 41 & 40.6 \\
Hispanic & 19 & 18.8 \\
Non-Hispanic White & 28 & 27.7 \\
Other & 13 & 12.9 \\
Unknown & & \\
Parent primary language & 64 & 63.4 \\
English & 36 & 35.6 \\
Non-English & 1 & 1.0 \\
Unknown & & \\
Medical insurance type & 68 & 67.3 \\
Public & 33 & 32.7 \\
Private & & \\
Hydronephrosis side & 71 & 70.3 \\
Unilateral & 30 & 29.7 \\
Bilateral & & 35.1 \\
Prenatally-diagnosed hydronephrosis severity (among n=131 kidneys) \\
Mild & 47 & 35.9 \\
Moderate & 38 & 29.0 \\
Severe & 46 & \\
\hline
\end{tabular}

Table 2 Number and rate of kidneys requiring surgical intervention among 131 kidneys diagnosed prenatally with mild, moderate, and severe hydronephrosis

\begin{tabular}{llll}
\hline $\begin{array}{l}\text { Hydronephrosis } \\
\text { severity }\end{array}$ & Total $(\mathrm{n})$ & $\begin{array}{l}\text { Surgical } \\
\text { intervention (n) }\end{array}$ & $\begin{array}{l}\text { Surgical } \\
\text { intervention } \\
(\%)\end{array}$ \\
\hline Mild & 47 & 4 & $8.5 \%$ \\
Moderate & 38 & 10 & $26.3 \%$ \\
Severe & 46 & 30 & $65.2 \%$ \\
Total & 131 & 44 & $33.6 \%$ \\
\hline
\end{tabular}

with mild HN (Fig. 1). Patients with severe $\mathrm{HN}$ were 12.2 times more likely to require surgery than patients with mild HN (95\% CI 6.1-24.4; $p<0.001$ ), and 2.9 times more likely to require surgery than patients with moderate $\mathrm{HN}$ (95\% CI 1.5-5.3; $p=0.003)$. Additionally, patients with moderate $\mathrm{HN}$ were 4.3 times more likely to require surgery than patients with mild HN (95\% CI 1.5-12.9; $p=0.01$ ). There was no significant difference in probability of receiving surgery by patient gender, race/ethnicity, primary language, or medical insurance type. Additionally, there was no significant difference in the probability 


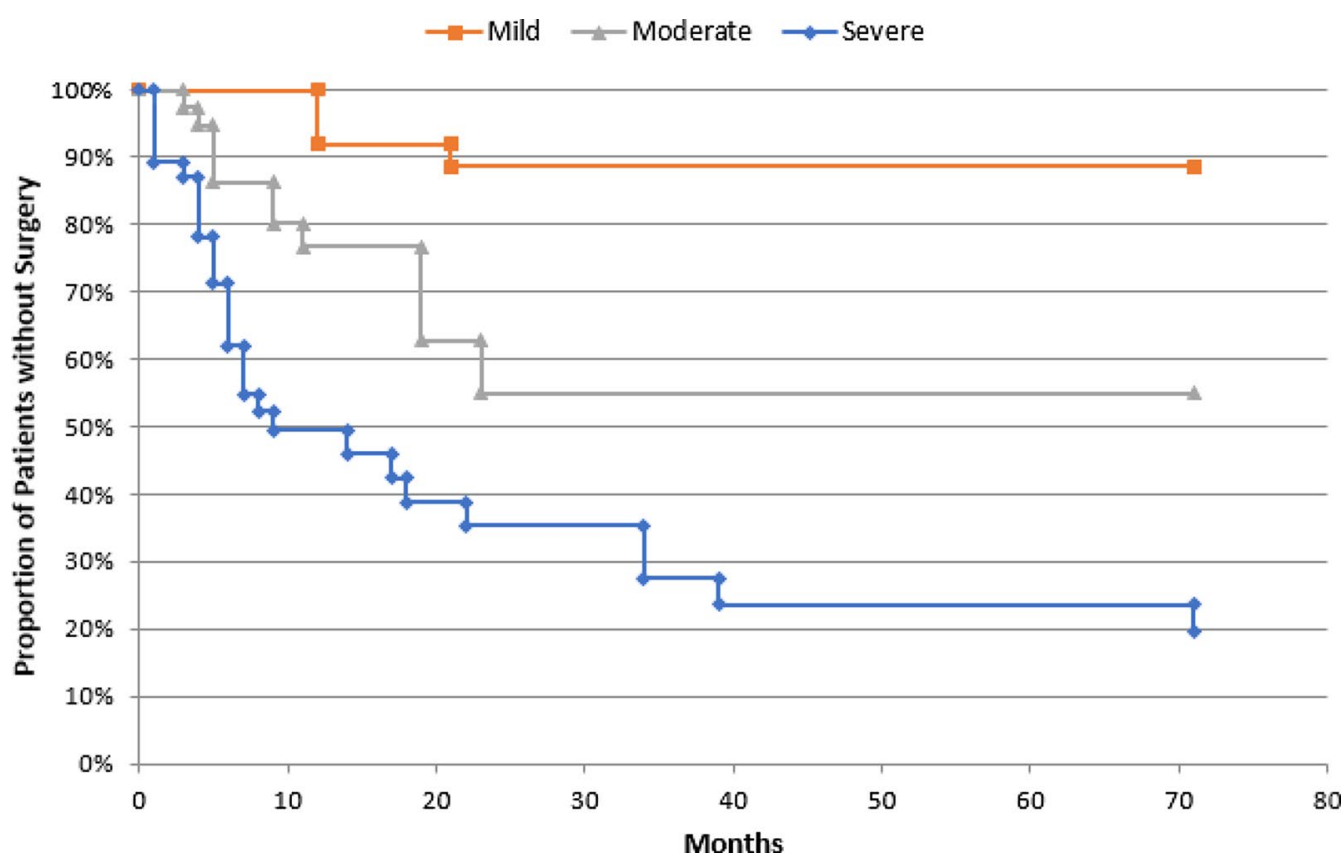

Fig. 1 Kaplan-Meier curves indicating surgery-free survival time in months, by prenatally-diagnosed hydronephrosis severity type

of surgical intervention among patients with evidence of VUR, compared to those with no evidence of VUR $(45.5 \%$ vs. $43.8 \%$; $\mathrm{X}^{2} p=0.72$ ).

\section{Patients with mild prenatally diagnosed hydronephrosis}

Among the 131 kidneys included in the study, 47 (35.9\%) were prenatally diagnosed with mild HN. Most patients $(74.5 \%, \mathrm{n}=35)$ who were diagnosed with mild $\mathrm{HN}$ had transient etiologies that self-resolved (Table 3; Fig. 2). Overall, four patients $(8.5 \%)$ with mild $\mathrm{HN}$ required surgery for $\mathrm{HN}$ postnatally, which occurred at a median age of 11.8 months (IQR 11.7-14.1 months; Fig. 1). At the time of analysis, 20 kidneys with mild $\mathrm{HN}$ had spontaneously resolved, and 23 were still being followed with serial ultrasound. The Kaplan-Meier estimate indicates that $8.1 \%$ (95\% CI $0.0-16.9 \%$ ) of patients with mild HN require surgery by one year of age, and $11.4 \%$ (95\% CI $0.8-22.0 \%$ ) require surgery by six years of age (Fig. 1 ). $\mathrm{HN}$-related diagnoses among patients who required surgery included UPJO in three cases, and ureterocele in one case (Table 3; Fig. 2). Three of these patients underwent pyeloplasty, and one patient underwent ureteral reimplantation, ureterocele incision, and bladder neck reconstruction (Table 3). In addition to transient etiologies, other etiologies that did not require surgical intervention among patients with mild HN included partial UPJO that did not meet the requirements for surgical intervention in five kidneys, VUR in three patients, and ureterocele in one patient.

\section{Patients with moderate prenatally diagnosed hydronephrosis}

A total of 38 kidneys (29.0\%) were diagnosed with moderate prenatal HN, of which $10(26.3 \%)$ required surgery (Table 2). Four kidneys with moderate HN spontaneously resolved, and the majority $(n=24)$ were still being followed for moderate $\mathrm{HN}$ at the time of data analysis. The Kaplan-Meier estimate indicates that $23.4 \%$ (95\% CI 10.2-36.6\%) of patients with moderate $\mathrm{HN}$ require surgery by one year of age, and $45.4 \%$ (95\% CI 22.5-68.3\%) may require surgery by six years of age (Fig. 1). Patients with moderate $\mathrm{HN}$ who required surgery were a median of 6.6 months old at the time of surgery (IQR 4.2-16.4 months). The majority of patients who required surgery had UPJO $(n=7 ; 70 \%$ of patients who underwent surgery; Table 3; Fig. 2). Additionally, one patient who required surgery had a ureterocele, and two had VUR. Most patients $(\mathrm{n}=7)$ who required surgery underwent pyeloplasty, two patients underwent ureteral reimplantations, and one patient each underwent a ureteroureterostomy and a nephroureterectomy (Table 3). Patients who did not require surgery most frequently had a transient etiology of their $\operatorname{HN}(n=19$; Table 3; Fig. 2). Additionally, among patients who did not require surgical intervention, six had UPJO not meeting the requirements for surgical intervention, three had VUR, and one had a ureterocele. 
Table 3 HN-related diagnoses, number of patients who required surgery, and surgical interventions among patients with mild, moderate, and severe prenatally-diagnosed HN

\begin{tabular}{|c|c|c|c|c|c|}
\hline \multirow[t]{2}{*}{ HN severity } & \multirow[t]{2}{*}{ HN-related diagnoses* } & \multirow{2}{*}{$\begin{array}{l}\text { Total kidneys } \\
\text { n }\end{array}$} & \multirow{2}{*}{$\begin{array}{l}\text { Kidneys requiring } \\
\text { surgery } \\
n\end{array}$} & \multicolumn{2}{|l|}{ Surgical intervention } \\
\hline & & & & Surgery type & $\mathrm{n}$ \\
\hline \multirow[t]{5}{*}{ Mild } & UPJ obstruction & 8 & 3 & Pyeloplasty & 3 \\
\hline & Ureterocele & 2 & 1 & $\begin{array}{l}\text { Ureterocele incision, ureteral reimplantation, } \\
\text { bladder neck reconstruction }\end{array}$ & 1 \\
\hline & VUR & 3 & 0 & - & - \\
\hline & Transient & 35 & - & - & - \\
\hline & Total & 47 & 4 & - & - \\
\hline \multirow[t]{6}{*}{ Moderate } & UPJ obstruction & 13 & 7 & Pyeloplasty & 7 \\
\hline & & & & Ureteroureterostomy & 1 \\
\hline & VUR & 5 & 2 & Ureteral reimplantation & 2 \\
\hline & Ureterocele & 2 & 1 & Nephroureterectomy & 1 \\
\hline & Transient & 19 & - & - & - \\
\hline & Total & 38 & 10 & - & - \\
\hline \multirow[t]{12}{*}{ Severe } & UPJ obstruction & 25 & 21 & Pyeloplasty & 17 \\
\hline & & & & Partial nephrectomy & 3 \\
\hline & & & & Cutaneous ureterostomy & 1 \\
\hline & & & & Vesicostomy & 1 \\
\hline & PUV & 4 & 4 & Valve ablation & 4 \\
\hline & Ureterocele & 4 & 2 & Ureterocele incision & 2 \\
\hline & VUR & 3 & 3 & Vesicostomy & 2 \\
\hline & & & & Ureteral reimplantation & 1 \\
\hline & UVJ obstruction & 2 & 2 & Ureteral reimplantation & 2 \\
\hline & Megaureter & 1 & 1 & Circumcision & 1 \\
\hline & Transient & 10 & - & - & - \\
\hline & Total & 46 & 30 & - & - \\
\hline
\end{tabular}

*Patients may have more than 1 diagnosis and may have undergone more than 1 type of surgical intervention

\section{Patients with severe prenatally diagnosed hydronephrosis}

Kidneys with severe HN accounted for $35.1 \%$ of kidneys included in the study $(n=46)$. Postnatal surgical intervention was required among $65.2 \%(n=30)$ of kidneys with severe $\mathrm{HN}$, and patients received surgery at a median of 5.4 months of age (3.7-12.4 months; Fig. 1). Of the remaining kidneys, six self-resolved and 10 were being followed conservatively. The Kaplan-Meier estimate indicates that 50.5\% (95\% CI 36.3-64.8\%) of patients with severe $\mathrm{HN}$ require surgery by one year of age, and $80.3 \%$ (95\% CI 66.0-94.7\%) may require surgery by six years of age (Fig. 1). UPJO remained the most common HN-related diagnosis among patients with severe $\mathrm{HN}$ who underwent surgery, occurring in $70.0 \%(\mathrm{n}=21)$ of these patients (Table 3; Fig. 2). Other HN-related diagnoses among patients who underwent surgery included posterior urethral valves (PUV), ureterocele, ureterovesical junction obstruction (UVJO), VUR, and megaureter. Among the 30 kidneys requiring surgery, 17 underwent pyeloplasty (Table 3). Additional surgical interventions included valve ablation, partial nephrectomy, cutaneous ureterostomy, vesicostomy, ureterocele incision, ureteral reimplantation, and circumcision for recurrent urinary tract infections (Table 3). Among the 16 patients with severe prenatal HN who did not require surgical intervention, diagnoses included transient etiologies $(n=10)$, UPJO not meeting the requirements for surgical intervention $(n=4)$, and ureterocele $(n=2)$.

\section{Discussion}

Prenatal $\mathrm{HN}$ is the most common prenatal abnormality detected on ultrasound and is a common referral to pediatric urologists [1]. How this $\mathrm{HN}$ can be managed depends on the severity of $\mathrm{HN}$ and concurrent findings that are identified in postnatal evaluation, generally by VCUG and renal US $[4,6]$. The aim of this study was to stratify the proportion of patients with mild, moderate, and severe $\mathrm{HN}$ who eventually require surgical intervention in order to gain greater insight into how to adequately counsel families prenatally on the management of HN. There was a relatively homogenous spread of the severity of $\mathrm{HN}$ in this cohort of 101 patients $(35.9 \%$ 


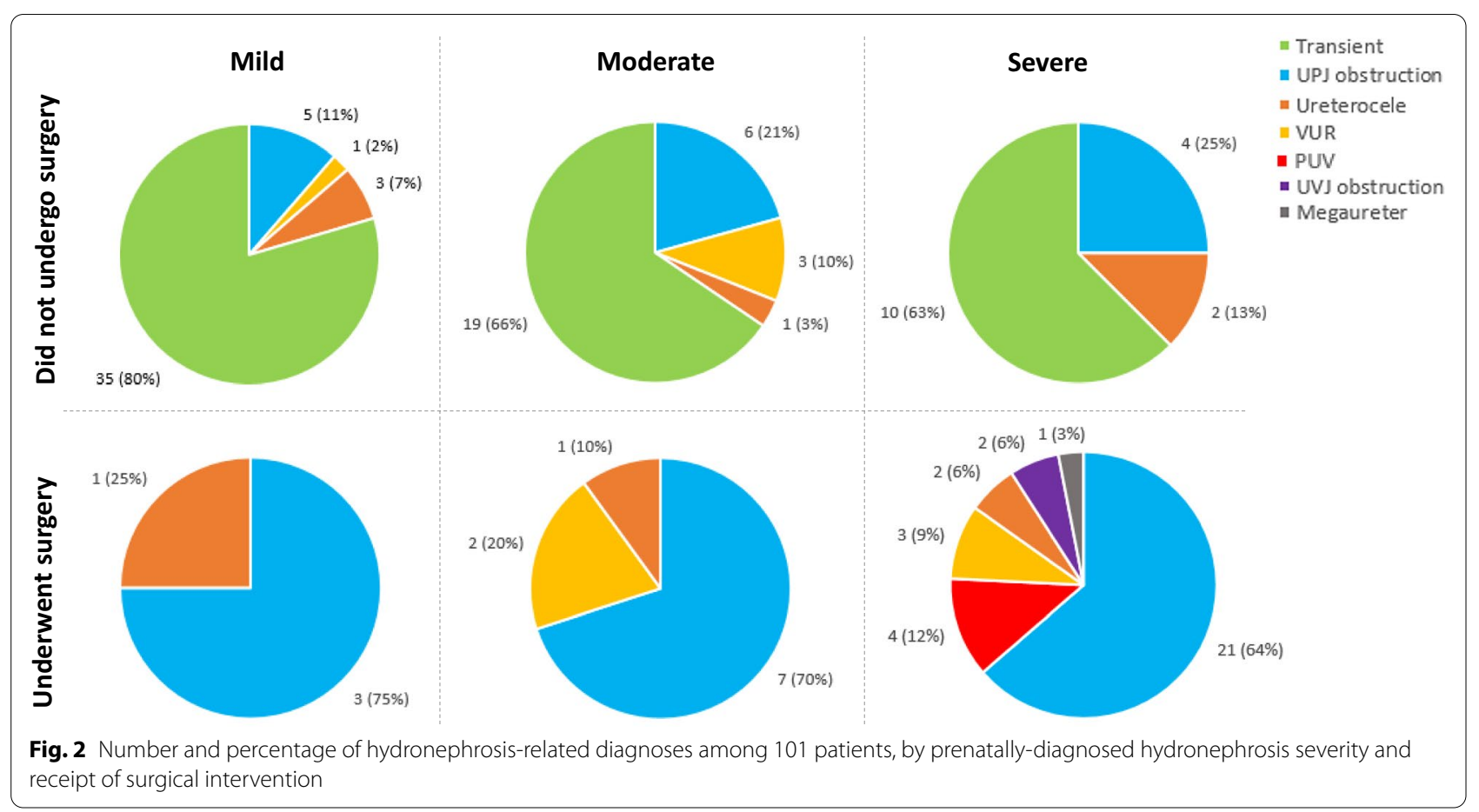

mild, $29.0 \%$ moderate, and $35.1 \%$ severe). It has been previously reported that $35-50 \%$ of patients with antenatal HN will have no HN on postnatal renal US [6]. The etiology of $\mathrm{HN}$ in our cohort also similarly reflected findings from previous studies. The most common pathological finding in our cohort was also UPJO, but the most common cause overall of $\mathrm{HN}$ was transient $\mathrm{HN}$ [6].

\section{Patients with mild prenatally diagnosed hydronephrosis}

Among patients with mild prenatal $\mathrm{HN}, 74.5 \%$ had a transient etiology that resolved postpartum, none of whom had surgical intervention. As with previously reported studies, patients with transient HN determined to no longer be present postnatally may not warrant close urologic follow-up after a negative ultrasound result [4]. However, $8.5 \%$ of patients with mild prenatal $\mathrm{HN}$ did require surgical intervention, suggesting that postnatal follow-up of patients diagnosed with mild prenatal $\mathrm{HN}$ is warranted.

\section{Patients with moderate prenatally diagnosed hydronephrosis}

As with the patients with mild HN, those with moderate $\mathrm{HN}$ who were determined to have transient $\mathrm{HN}$ did not require surgical intervention. While transient $\mathrm{HN}$ was less common in patients with moderate versus mild prenatal $\mathrm{HN}$, it still occurred in half of all patients diagnosed with moderate prenatal HN. Approximately one quarter of all kidneys prenatally diagnosed with moderate
$\mathrm{HN}$ required surgery. Time to surgery in this cohort was notable. The median time to surgery for patients with moderate HN was 6.6 months, compared to 11.8 months in those with mild $\mathrm{HN}$ and 5.4 months in those with severe HN. Liu et al. noted that the rate of pathology in those with moderate prenatal $\mathrm{HN}$ was $45.1 \%$ (25.3-66.6) [15]. This raises the question of why those with moderate $\mathrm{HN}$ were likely to undergo intervention around the same as those with more severe findings. While fewer patients with moderate $\mathrm{HN}$ underwent surgery overall compared to those with severe $\mathrm{HN}$, one must consider what prompts practitioners to consider intervention particularly in this moderate group.

\section{Patients with severe prenatally diagnosed hydronephrosis} Like those in the mild and moderate cohorts, those patients with severe $\mathrm{HN}$ who were determined to have transient HN did not undergo surgical intervention. As one would expect, the patients with severe prenatal $\mathrm{HN}$ were significantly more likely to require some form of surgical intervention, which occurred at the earliest median time, as compared to mild and moderate cohorts. Among all kidneys with severe prenatal HN, almost 50\% required surgical intervention due to UPJO. Surgical interventions required for $\mathrm{HN}$-related diagnoses were also broader than patients with mild or moderate $\mathrm{HN}$, including valve ablation, vesicostomy, cutaneous ureterostomy, partial nephrectomy, and circumcision for recurrent urinary tract infection in some cases. Data from 
this study suggest that patients with severe prenatal HN require close postnatal follow-up and may require surgery within the first six months of life.

\section{Limitations}

This study is not without its limitations. Due to the retrospective nature of this study, classifications of $\mathrm{HN}$ severity were not standardized; while some providers used SFU grades, others used descriptions of "mild," "moderate," or "severe" to diagnose prenatal HN severity. Additionally, with only 101 patients in the cohort, our study is underpowered to be able to adequately stratify the severity of $\mathrm{HN}$ with the type of surgical procedure; however, it was possible to assess the association between increased $\mathrm{HN}$ severity and increased risk of surgical intervention of any type. The decision to recommend surgery was based on the patients' clinical characteristics and the pediatric urologists' judgements, which may vary depending on the surgeon. Additionally, as the median length of follow-up time in this study was 1.2 years, the Kaplan-Meier estimator for surgery-free survival through six years may be unreliable as it relies on data from few participants. The broad confidence intervals provided reflect the uncertainty of the six-year Kaplan-Meier estimates. For this reason, the six-year surgery-free survival estimate should be considered with caution, and the Kaplan-Meier estimator for surgery-free survival through one year is more reliable. Furthermore, assessment of outcomes among patients with prenatal $\mathrm{HN}$ in a multi-institutional setting would hold more impact in being able to determine how to manage the equivocal cases of prenatal $\mathrm{HN}$.

\section{Conclusions}

The experience from this maternal fetal health center provides further reassurance that patients with transient $\mathrm{HN}$ will not likely require surgical intervention in the future, despite severity. However, regardless of the nature of $\mathrm{HN}$, those with moderate and severe $\mathrm{HN}$ do require further urologic work up beyond postnatal renal US to evaluate for potential pathologies and prevent infection or loss of renal function. Additionally, it reiterates the importance of prenatal counseling for close postnatal follow-up no matter the degree of prenatal hydronephrosis as it is difficult to predict the likelihood of surgery or resolution of $\mathrm{HN}$ on prenatal ultrasound alone. Even the mild form of prenatal HN may have pathology that requires surgical intervention. However, patients who are diagnosed with prenatal $\mathrm{HN}$ are significantly more likely to require surgical intervention and at an early age if they are found to have severe HN. Close follow-up and adequate family counseling in those with more severe $\mathrm{HN}$ prenatally are warranted.

\section{Abbreviations}

AP: Anteroposterior; Cl: Confidence Interval; HN: Hydronephrosis; HR: Hazard Ratio; IQR: Interquartile Range; PUV: Posterior Urethral Valves; SFU: Society for Fetal Urology; UPJO: Ureteropelvic Junction Obstruction; UVJO: Ureterovesical Junction Obstruction; US: Ultrasonography; UTI: Urinary Tract Infection; VCUG: Voiding Cystourethrogram; VUR: Vesicoureteral Reflux.

\section{Acknowledgements}

Not applicable.

\section{Authors' contributions}

ZB collected and analyzed data and wrote the manuscript. AH assisted with data analysis and writing the manuscript. MT and DE assisted with data collection and analysis. CD assisted with data collection and analysis and writing the manuscript. AC, SS, and RD helped to conceptualize the study design, saw patients, and reviewed and edited the manuscript. EV oversaw the project, conceptualized the study, saw patients, and reviewed and edited the manuscript. All authors have read and approved the final manuscript.

\section{Funding}

This study is unfunded.

\section{Availability of data and materials}

In order to uphold participant confidentiality and adhere by the guidelines stipulated by our IRB, data will not be made publicly available.

\section{Declarations}

Ethics approval and consent to participate

This study was reviewed and approved by the Institutional Review Board at Children's Hospital Los Angeles (FWA0001914). The research team was approved by the IRB at our institution to access and use our institution's electronic medical record for the purposes of this study. Considering the retrospective nature of this study and appropriate protocols to protect participant confidentiality, the IRB determined that informed consent was not required for this study.

\section{Consent for publication}

Not applicable.

\section{Competing interests}

The authors have no financial or non-financial competing interests to be declared.

\section{Author details \\ ${ }^{1}$ Division of Urology, Children's Hospital Los Angeles, Los Angeles, CA, USA. ${ }^{2}$ Keck School of Medicine of Medicine of USC, University of Southern Califor- nia, Los Angeles, CA, USA. ${ }^{3}$ Thomas J. Long School of Pharmacy, University of the Pacific, Stockton, CA, USA.}

Received: 10 November 2020 Accepted: 24 March 2021

Published online: 07 April 2021

\footnotetext{
References

1. Yalcinkaya F, Ozcakar ZB. Management of antenatal hydronephrosis Pediatr Nephrol. 2019. https://doi.org/10.1007/s00467-019-04420-6.

2. Lee RS, Cendron M, Kinnamon DD, Nguyen HT. Antenatal hydronephrosis as a predictor of postnatal outcome: A meta-analysis. Pediatrics. 2006;118(2):586-93. https://doi.org/10.1542/peds.2006-0120.

3. Arnaud A, Hossini SL, de Lara ST, Dobremez E, Chateil J, Harper L. Managing children with hydronephrosis: Common pitfall during ultrasound follow-up to remember. Arch Dis Child. 2019. https://doi.org/10.1136/ archdischild-2019-317386.

4. Kohno M, Ogawa T, Kojima Y, Sakoda A, Johnin K, Sugita Y, Nakane A, Noguchi M, Moriya K, Hattori M, Hayashi Y, Kubota M. Pediatric congenital hydronephrosis (ureteropelvic junction obstruction): Medical management guide. Int J Urol. 2020;27:369-76.
} 
5. Braga LH, Mijovic H, Farrokhyar F, Pemberton J, DeMaria J, Lorenzo AJ. Antibiotic prophylaxis for urinary tract infections in antenatal hydronephrosis. Pediatrics. 2013;131(1):e251-61. https://doi.org/10.1542/peds. 2012-1870.

6. Woodward M, Frank D. Postnatal management of antenatal hydronephrosis. BJU Int. 2002. https://doi.org/10.1046/j.1464-4096.2001.woodw ard.2578.x.

7. Passoni NM, Peters CA. Managing ureteropelvic junction obstruction in the young infant. Front Pediatr. 2020;8:242

8. Chertin B, Pollack A, Koulikov D, Rabinowitz R, Hain D, Hadas-Halpren I, Farkas A. Conservative treatment of ureteropelvic junction obstruction in children with antenatal diagnosis of hydronephrosis: Lessons learned after 16 years of follow-up. Eur Urol. 2006;49(4):734-9. https://doi.org/10. 1016/j.eururo.2006.01.046

9. Sadeghi-Bojd S, Kajbafzadeh A, Ansari-Moghadam A, Rashidi S. Postnatal evaluation and outcome of prenatal hydronephrosis. Iran J Pediatr. 2016;26(2):e3667.

10. Yang $Y$, Hou Y, Niu ZB, Wang CL. Long-term follow-up and management of prenatally detected, isolated hydronephrosis. J Pediatr Surg. 2010;45(8):P1701-1706.
11. Ulman I, Jayanthi VR, Koff SA. The long-term followup of newborns with severe unilateral hydronephrosis initially treated nonoperatively. J Urol. 2000;164(3):1101-5.

12. Arena S, Chimenz R, Antonelli E, Peri FM, Romeo P, et al. A long-term follow-up in conservative management of unilateral ureteropelvic junction obstruction with poor drainage and good renal function. Eur J Pediatr. 2018;177:1761-5.

13. Leong SY, Afolabi TM, Tsu LV. The management of antenatal hydronephrosis U.S. Pharmacist. 2016:41(5):112-6.

14. Sung J, Skoog S. Surgical management of vesicoureteral reflux in children. Pediatr Nephrol. 2012;27(4):551-61. https://doi.org/10.1007/ s00467-011-1933-7.

15. Liu DB, Armstrong WR 3rd, Maizels M. Hydronephrosis: prenatal and postnatal evaluation and management. Clin Perinatol. 2014;41(3):661-78. https://doi.org/10.1016/j.clp.2014.05.013.

\section{Publisher's Note}

Springer Nature remains neutral with regard to jurisdictional claims in published maps and institutional affiliations.
Ready to submit your research? Choose BMC and benefit from:

- fast, convenient online submission

- thorough peer review by experienced researchers in your field

- rapid publication on acceptance

- support for research data, including large and complex data types

- gold Open Access which fosters wider collaboration and increased citations

- maximum visibility for your research: over $100 \mathrm{M}$ website views per year

At BMC, research is always in progress.

Learn more biomedcentral.com/submissions 